\title{
COMMUTATIVE RINGS WHOSE MATRIX RINGS ARE BAER RINGS
}

\author{
CLEON R. YOHE
}

A ring $R$ with unit element is a Baer ring if every left annihilator in $R$ has the form $R e$, where $e$ is an idempotent element. K. G. Wolfson has proven [3, Corollary 15], that if $R$ is a Prüfer ring (a commutative integral domain in which every finitely generated ideal is invertible) then the ring of endomorphisms of a finitely generated free module over $R$ is a Baer ring. In this note we view these endomorphism rings as the matrix rings $R_{n}$ with which they are isomorphic, and show that under the rather modest assumption that $R$ have descending chain condition on annihilators, the converse of this result holds. It is also shown that in this case all matrix rings over $R$ are Baer rings if any one of them is.

THEOREM. Let $R$ be a commutative ring with unit element and descending chain condition on annihilators. Then the following are equivalent:

(1) $R_{n}$ is a Baer ring for every $n \geqq 2$.

(2) $R_{k}$ is a Baer ring for some particular $k \geqq 2$.

(3) $R$ is a finite direct sum of Prüfer rings.

Proof. To show that (3) implies (1), let $R=R_{1} \oplus \cdots \oplus R_{t}$, where the $R_{i}$ are Prüfer rings. Then since the matrix ring $R_{n}=\left(R_{1}\right)_{n} \oplus \ldots$ $\oplus\left(R_{t}\right)_{n}$ and finite direct sums of Baer rings are Baer rings, this reduces to Wolfson's result. That (1) implies (2) is obvious.

To establish that (2) implies (3), suppose that $R_{k}$ is a Baer ring for some specific integer $k \geqq 2$. Define a matrix $\left(e_{i j}\right) \in R_{k}$ by $e_{11}=e_{22}=1$, $e_{i j}=0$ otherwise. This matrix is idempotent, and therefore $\left(e_{i j}\right) R_{k}\left(e_{i j}\right)$ is a Baer ring [2, Theorem 2, p. 3]. Since this subring of $R_{k}$ is isomorphic to $R_{2}$, we may assume from the outset that $k=2$.

We wish to show first that $R$ is a direct sum of integral domains, and so it may be assumed that $R$ contains zero-divisors. Let $A \neq(0)$ be a minimal annihilator in $R$. This ideal exists because $R$ is not a domain. Since the center of a Baer ring is again a Baer ring [2, Corollary to Theorem 3, p. 4], $A=R e$, where $e^{2}=e \neq 0$. Suppose that $a, b \in A, b \neq 0$ and $a b=0$. Then ann $(a) \cap A \neq(0)$, so by the minimality of $A, A \subseteq \operatorname{ann}(a)$, whence $a A=(0)$. Putting $a=r e$ for an appropriate $r \in R$, we have $R r e=r e R e=a A=(0)$, so $a=r e=0$ and $A$ is an integral domain. Let $A_{1}=A, B_{1}=R(1-e)$; clearly $R=A_{1} \oplus B_{1}$. The ring $B_{1}$ also has descending chain condition on annihilators. If $B_{1}$ has divisors

Received by the editors September 5, 1968. 
of zero, then since the matrix ring $R_{2}=\left(A_{1}\right)_{2} \oplus\left(B_{1}\right)_{2}$ and a direct summand of a Baer ring is a Baer ring [2, Corollary to Theorem 2, p. 3], we may repeat the process on $B_{1}$ to get $B_{1}=A_{2} \oplus B_{2}$ where $A_{2}$ is an integral domain. Then $R=A_{1} \oplus A_{2} \oplus B_{2}$. Continuing in this way, one obtains a descending chain of annihilators $\left\{B_{i}\right\}$, and thus by hypothesis there must exist an integer $t$ such that $B_{t}=B_{t+1}=\cdots$. This can only mean that $B_{t}$ is an integral domain, since otherwise the procedure could be continued. Hence $R=A_{1} \oplus \cdots \oplus A_{t} \oplus B_{t}$ is a direct sum of integral domains.

Since it is clear from the facts quoted above that each of these summands has a 2-by-2 matrix ring which is a Baer ring, we shall have completed the proof of the theorem if we show that whenever $R$ is a commutative integral domain such that $R_{2}$ is a Baer ring, then $R$ is a Prüfer ring. Let $R$ be such a domain.

Suppose that $P$ is any prime ideal of $R$, and that $R_{P}$ is the localization of $R$ at $P$. Let $T \in\left(R_{P}\right)_{2}$ be a zero-divisor in the 2 -by-2 matrix ring over $R_{P}$. Write the entries of $T$ over a common denominator $a$, so that $T=\left(a_{i j} / a\right), a_{i j}, a \in R, a \neq 0$. It is clear that the matrix $\left(a_{i j}\right)$ is a zero-divisor of $R_{2}$, and hence by hypothesis there is an idempotent $\left(e_{i j}\right) \neq 0$ such that $\left(e_{i j}\right)\left(a_{i j}\right)=0$. Then in $\left(R_{P}\right)_{2},\left(e_{i j}\right) T=0$, so $\left(R_{P}\right)_{2}$ has the property that the left annihilator of any zero-divisor contains a nonzero idempotent. Let $a$ and $b$ be any two nonzero elements of $R_{P}$. Form the matrix $\left(a_{i j}\right) \in\left(R_{P}\right)_{2}$ which has entries $a_{11}=a, a_{21}=b, a_{12}$ $=a_{22}=0$; then $\left(a_{i j}\right)$ is a zero-divisor, so there exists an idempotent $\left(e_{i j}\right) \neq 0$ with $\left(e_{i j}\right)\left(a_{i j}\right) \neq 0$. Notice that not all of the $e_{i j}$ can be in $P_{P}$, the maximal ideal of $R_{P}$, for the Jacobson radical of $\left(R_{P}\right)_{2}$ is exactly the set of matrices all of whose entries come from $P_{P}$, and the radical of a ring cannot contain nonzero idempotents. The equality $\left(e_{i j}\right)\left(a_{i j}\right)$ $=0$ yields the two equations $e_{11} a+e_{12} b=0, e_{21} a+e_{22} b=0$. But one of the $e_{i j}$ is not in $P_{P}$ and therefore is a unit of $R_{P}$. Multiplication of the appropriate one of these two equations by the inverse of this $e_{i j}$ displays one of $a$ or $b$ as a multiple of the other.

Hence for any prime ideal $P, R_{P}$ has the property that whenever $a$ and $b$ are in $R_{P}$ and are not zero, then one of them is a multiple of the other, i.e. $R_{P}$ is a valuation ring. It is well known [1, Exercise 12, p. 93] that an integral domain with this property has every finitely generated ideal invertible, and therefore we have shown that $R$ is a Prüfer ring.

Observing that a commutative noetherian ring has descending chain condition on annihilators and that a noetherian Prüfer ring is a Dedekind domain, we obtain an interesting special case of the theorem. 
COROLlARY. Let $R$ be a commutative noetherian ring. Then every $R_{n}$ is a Baer ring if and only if $R$ is a direct sum of Dedekind domains.

Acknowledgement. This research was supported in part by National Science Foundation grant number GP 7175.

\section{REFERENCES}

1. N. Bourbaki, Algèbre commutative, Chapitre VII, Hermann, Paris, 1965.

2. I. Kaplansky, Rings of operators (mimeographed), University of Chicago, 1955.

3. K. G. Wolfson, Baer rings of endomorphisms, Math. Ann. 143 (1961), 19-28.

WASHINGTON UNIVERSITY 